ESJ Natural/Life/Medical Sciences

\title{
Ethnozoologie De Balearica Pavonina (Grue Couronnée) Dans La Vallée Du Fleuve Niger: Cas Du Barrage De Kandadji
}

\author{
Soumaïla Hassane Hamani \\ Abdourahamane Illiassou
}

Université de Diffa, Faculté des Sciences Agronomiques, Département de production Animale, Diffa

\section{Youssoufa Issiaka}

Université Dan Dicko Dankoulodo de Maradi, Faculté d’Agronomie et des Sciences de l'Environnement, Département Génie Rural et Eaux et Forêts, Maradi, Niger

\section{Ali Mahamane}

Université Abdou Moumouni de Niamey, Faculté des Sciences et Techniques, Département de Biologie, Niamey, Niger

Doi:10.19044/esj.2021.v17n3p150

Submitted: 14 August 2020

Accepted: 16 March 2020

Published: 31 January 2021
Copyright 2021 Author(s)

Under Creative Commons BY-NC-ND

4.0 OPEN ACCESS

Cite As:

Hassane Hamani S., Illiassou A., Issiaka Y. \& Mahamane A. (2021). Ethnozoologie De Balearica Pavonina (Grue Couronnée) Dans La Vallée Du Fleuve Niger: Cas Du Barrage De Kandadji. European Scientific Journal, ESJ, 17(3), 150.

https://doi.org/10.19044/esj.2021.v17n3p150

\section{Résumé}

La présente étude menée dans la zone du barrage de Kandadji a pour but principal de caractériser les différents aspects biologiques et écologiques de l'espèce Balearica pavonina dans la zone. La méthode de collecte des données s'est basée sur la recherche documentaire et aux enquêtes ethnozoologiques auprès de 311 personnes par des entretiens individuels, du 15 Septembre au 31 Octobre 2018. Les données collectées ont été analysées par le logiciel Excel. Quelques éléments ethnozoologiques ont été caractérisés. La Grue couronnée est un oiseau échassier dont le plumage du corps et du cou sont gris foncés ou noirs. Elle fréquente principalement les zones humides et herbeuses. Cette espèce est aperçue le matin par 54,34\% de la population et le soir par 33,11\%. La Grue couronnée est aperçue il y a un jour par 30,54\% de la population et une semaine par 34,72\%. Elle est rencontrée toutes les saisons de l'année à des colonies d'environ 10 individus. L'étude a 
montré que la sècheresse est la principale menace naturelle avec $87,13 \%$ des sondages. Ensuite, la destruction de l'habitat et la chasse constituent des menaces anthropiques respectivement avec $38,26 \%$ et $36,01 \%$ des sondages. Sa nourriture est constituée des insectes (33,11\%) et graines (51,76\%). Les œufs de B. pavonina sont blancs $(87,13)$ et de grande de taille $(81,99 \%)$. Cette espèce pond plus de deux œufs (65,91\%) dans les herbes (64,95\%). Le temps d'éclosion des œufs est de un mois (83,92\%) selon les sondages. La Grue couronnée figure parmi les espèces en voie de disparition. Cette étude vise à contribuer à la préservation de cette ressource animale.

Mots-clés: Ethnozoologie, Balearica Pavonina, Barrage De Kandadji, Régime Alimentaire

\title{
Ethnozoology Of Balearica Pavonina (Crowned Crane) In The Valley Of The Niger River: Case Of The Kandadji Dam
}

\section{Soumaïla Hassane Hamani Abdourahamane Illiassou}

Université de Diffa, Faculté des Sciences Agronomiques, Département de production Animale, Diffa

\section{Youssoufa Issiaka}

Université Dan Dicko Dankoulodo de Maradi, Faculté d'Agronomie et des Sciences de l'Environnement, Département Génie Rural et Eaux et Forêts, Maradi, Niger

\section{Ali Mahamane}

Université Abdou Moumouni de Niamey, Faculté des Sciences et Techniques, Département de Biologie, Niamey, Niger

\begin{abstract}
This study, undertaken in the zone of the Kandadji dam aimed to characterize the various biological and ecological aspects of the Balearica pavanina (crowned crane ) in the zone. Thedata collection was based on the information retrieval and the ethnozoologic investigations based on questionnaires administrated to 311 people from September, $15^{\text {th }}$ to October $31^{\text {st }} 2018$. After an analysis of the data with the Excel software, someof the ethnozoologic elements were characterized. Hence, the crowned crane is a wader whose body plumage and neck are dark gray or black. It is mainly found in wetlands and grassland. This species is seen in the morning by $54,34 \%$ of the population and the evening by $33,11 \%$. The sighting frequency of the crowned Crane varies from a day ago by $30,54 \%$ of the population to a week by $34,72 \%$. It is found in every season of the year in colonies of approximately
\end{abstract}


10 individuals. The study also showed that the drought is the principal natural threat with $87,13 \%$ of the surveys, followed by habitat destruction and hunting as anthropic threats with $38,26 \%$ and $36,01 \%$ of the survey, respectively. The crowned crane feeds on insects (33,11\%) and seeds $(51,76 \%)$. The eggs of $B$. pavonina are white $(87,13)$ and of large size $(81,99 \%)$. This species lays more than two eggs 65,91\%) in the grasses (64,95\%). The duration of blossoming of eggs is one month $(83,92 \%)$ according to the survey. The crowned crane figures among endangered speciesand this study will contribute to the safeguarde this animal resource.

Key words: Ethnozoology, Balearica pavonina, Kandadji dam, food mode, Ecology.

\section{Introduction}

Classées parmi les écosystèmes les plus productifs de la terre, les zones humides offrent d'immenses possibilités de développement durable (Issiaka, 2004). Cette importance se mesure par rapport à leurs fonctions, leurs valeurs et leurs attributs déterminants aussi bien pour l'équilibre de l'environnement que pour la production agricole (Fousseni, 2000 et Issiaka 2011). Nonobstant cela, ces écosystèmes constituent un habitat par excellence des oiseaux, des véritables indicateurs biologiques qui peuvent renseigner sur l'état et le bon fonctionnement de ces derniers (Soumaila, 2014). En effet, les oiseaux constituent un groupe zoologique aisément utilisable en matière de diagnostic écologique capable de déboucher sur des stratégies de conservation favorable à d'autres espèces de la flore et de la faune (Sinsin et Kampman, 2010). Si les oiseaux d'eau disparaissent d'une zone humide, cela peut signifier dans certains cas que les ressources végétales ou animales se font rares y compris pour les populations humaines. Malgré leurs importances, les zones humides et oiseaux d'eau continuent à subir des menaces dans le monde. Parmi les effets de cette tendance dont pâtissent déjà les habitants, figurent le déclin des pêcheries, la pollution, la prolifération des algues toxiques et surtout l'érosion de la biodiversité (Issiaka, 2004). Au Niger, à l'instar des autres pays en développement, la sécheresse combinée à l'exploitation abusive par l'homme des ressources naturelles des zones humides est à la base de la détérioration des écosystèmes humides. Cette dernière rend vulnérable près de $90 \%$ de la population nigérienne qui dépend directement des ressources naturelles pour sa subsistance. La détérioration des zones humides du Niger a également considérablement contribué à la perte en leur biodiversité notamment l'avifaune. C'est le cas de la Grue Couronnée (Balearica pavonina), une espèce vulnérable selon la liste rouge de l'IUCN. Elle appartient à la famille de Guidea, l'une des familles d'oiseaux les plus menacées au monde. La population de l'espèce diminue par la dégradation de son habitat (Boere et $a l$, 
2006; Beilfuss et al, 2007; Harris et Mirande, 2013). La Grue couronnée fréquente principalement les zones humides et herbeuses, les grands marais, et on la voit également au bord des lacs ou des fleuves dans les eaux peu profondes. Afin de contrer la problématique hydrique, favoriser le développement du pays, renforcer la sécurité alimentaire et réduire la pauvreté sévère auquel le pays fait face, le gouvernement nigérien a adopté en 2002 le Programme "Kandadji" de Régénération des Écosystèmes et de Mise en valeur de la vallée du Niger (P-KRESMIN). Ce projet vise principalement à créer un ouvrage de régulation d'eau combinée à une production d'hydroélectricité sur le fleuve Niger, à côté du village de Kandadji. Pour compenser les effets potentiellement néfastes du barrage, une aire protégée intégrant les milieux humides, forêts rivulaires, milieux terrestres et milieux aquatiques est envisagée par le P-KRESMIN à travers son plan de gestion de la vie sauvage et des habitats naturels (Georges, 2015). C'est dans ce contexte que cette étude a été réalisée pour comprendre l'ethnobiologie et l'ethnoécologie de la Grue couronnée (Balearica pavonina) dans la zone du barrage de Kandadji en vue d'une gestion écologique durable de cette espèce et de son habitat. Il s'agit de faire la typologie de son habitat et de sa distribution spatio-temporelle; de déterminer son comportement alimentaire et son mode de reproduction.

\section{Matériel et méthodes}

\section{Milieu d'étude}

L'étude a été réalisée dans trois communes de la région de Tillabéry qui sont Ayerou $\left(00^{\circ} 92^{\prime} 01^{\prime \prime} \mathrm{E}\right.$ et $\left.14^{\circ} 71^{\prime} 38^{\prime \prime} \mathrm{N}\right)$, Dessa (Kandadji) $\left(00^{\circ} 99^{\prime} 10^{\prime \prime} \mathrm{E}\right.$ et $\left.14^{\circ} 61^{\prime} 34^{\prime}{ }^{\prime} \mathrm{N}\right)$ et Kokorou $\left(00^{\circ} 90^{\prime} 44^{\prime}\right.$ 'E et $\left.14^{\circ} 18^{\prime} 45^{\prime}{ }^{\prime} \mathrm{N}\right)$.

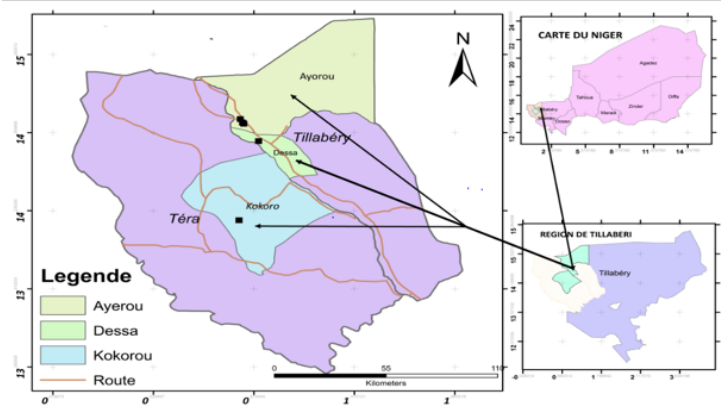

Figure 1.Carte de la zone d'étude

Les températures de la zone sont très élevées le jour, pouvant atteindre jusqu'à $45^{\circ} \mathrm{C}$ à l'ombre, puis basses la nuit; elles descendent parfois en dessous de $10^{\circ} \mathrm{C}$. La pluviométrie y est faible (en moyenne $240 \mathrm{~mm} / \mathrm{an}$ ) et est répartie très inégalement dans le temps et dans l'espace (Cissé, 2013). Les principales espèces floristiques observées au niveau du fleuve et des plaines inondables sont : Aeschynomene afraspera, Brachiaria mutica, Cyperus maculatus, 
Echinocloa colona, Echinocloa stagnina, Eragrostris pilosa, Ipomaea asarifolia, Nymphaea lotus, Oryza longistaminata, Panicum laetum, Polygonum senegalense et Vetiveria nigritana (Geesing et Djibo, 2006; Tecsult, 2006b).

La zone d'étude a une population estimée à 195288 habitants dont 38957 habitants pour la commune rurale de Dessa, 115934 habitants pour Kokorou et 40397 habitants pour Ayerou. Ces trois communes ont une superficie totale de $2629 \mathrm{~km} 2$. L'agriculture de subsistance, l'élevage, la pêche, le commerce et l'exploitation forestière représentent les activités de la zone (Cissé, 2013). L'agriculture se caractérise par la prédominance des céréales à double fonction (grain et sous-produits agricoles pour le cheptel) et par des cultures irriguées dominées par la riziculture et le maraîchage (Geesing et Djibo, 2006). La région est caractérisée par une couverture de matériaux meubles qui est relativement mince, de sorte que le substratum rocheux, situé près de la surface, y affleure sur de grandes étendues. Les ressources hydriques de la zone sont constituées du fleuve Niger et ces affluents dont la Sirba, le Dargol et le Goroual mais aussi par des mares comme Kokorou-Namga.

\section{Méthode de collecte des données}

Les recherchent documentaires ont permis d'élaborer une fiche d'enquête. Les enquêtes ethno-biologiques et ethnoécologiques ont été effectuées du 15 Septembre au 31 Octobre 2018, auprès des populations qui ont une connaissance et susceptibles de fournir des informations utiles sur la Grue couronnée. Au total 311 personnes ont été questionnées. Ces enquêtes ont été faites oralement par des entretiens individuels. Les personnes enquêtées ont été identifiées par des choix raisonnés et aléatoires, en tenant compte des critères ci-après :

- l'âge de l'enquêté permettant de tenir compte des différentes catégories d'âge pour un bon regroupement des connaissances liées à l'espèce.

- le sexe de l'enquêté pour une prise en compte effective des femmes et des hommes dans l'échantillon car les informations varient en fonction du sexe.

- l'ethnie de l'enquêté car les connaissances varient en fonction des ethnies.

En outre, des observations directes participatives sont également effectuées.

Les principales données collectées ont portées sur :

- La connaissance du nom de l'espèce dans les différentes langues de la population de ces sites ;

- La fréquence d'observation des Grues ; 
- La connaissance sur la typologie de l'habitat de l'espèce et leurs dynamiques spatiotemporelles;

La connaissance sur le régime alimentaire et le mode de reproduction de l'espèce

\section{Traitement des données}

Les informations collectées ont subi un traitement manuel et ont été codifiées avant leur intégration dans l’ordinateur pour être traitées par le logiciel Excel.

Minitab 14 est utilisé pour analyser la relation entre certains paramètres à travers le coefficient de corrélation de Pearson. Le seuil de signification de ces différents tests est $\alpha=0,05$.

\section{Résultats et discussion}

\section{Résultats}

\section{Profil des personnes enquêtées}

L'analyse du tableau 1 révèle que 243 hommes et 68 femmes ont été interrogés. Parmi les personnes interrogées $70,41 \%$ sont âgées de moins de 50 ans et $29,58 \%$ ont un âge supérieur ou égal à 50 ans. 97,42\% des enquêtés sont musulmans et 2,57\% sont chrétiens. L'ethnie la plus représentée est le zarma (80,38\%) (Tableau 1).

Tableau 1. Caractéristiques des personnes enquêtées

\begin{tabular}{|c|c|c|}
\hline Enquêtés & Valeurs & Pourcentage \\
\hline \multicolumn{3}{|c|}{ Sexe } \\
\hline Homme & 243 & $78,13 \%$ \\
\hline Femme & 68 & $21,86 \%$ \\
\hline \multicolumn{3}{|c|}{ âge } \\
\hline$<50$ ans & 219 & $70,41 \%$ \\
\hline$\geq 50$ ans & 92 & $29,58 \%$ \\
\hline \multicolumn{3}{|c|}{ Religion } \\
\hline Musulman & 303 & $97,42 \%$ \\
\hline Chrétienne & 8 & $2,57 \%$ \\
\hline \multicolumn{3}{|c|}{ Ethnie } \\
\hline Zarma & 250 & $80,38 \%$ \\
\hline Haoussa & 10 & $3,21 \%$ \\
\hline Touareg & 38 & $12,21 \%$ \\
\hline Peulh & 13 & $4,18 \%$ \\
\hline
\end{tabular}

\section{Typologie de l'habitat et dynamique spatio-temporelle de l'espèce}

\section{Entretien avec la population}

Les enquêtes ont révélé que 34,72\% des personnes ont rencontrées l'espèce il y'a seulement une semaine avant l'enquête, alors que seulement 1,61\% plus d'un an avant l'enquête. Selon 54,34\% des personnes enquêtées la 
Grue est plus fréquemment observable le matin que le soir ou à midi (Tableau 2).

Tableau 2. Fréquence de rencontre de la population avec l'espèce par période

\begin{tabular}{|l|l|l|l|l|l|l|l|l|}
\hline & \multicolumn{3}{|l}{ Dernière rencontre } & \multicolumn{3}{l|}{ Moment de rencontre } \\
\hline & $\begin{array}{l}01 \\
\text { jour }\end{array}$ & $\begin{array}{l}01 \\
\text { semaine }\end{array}$ & $\begin{array}{l}01 \\
\text { mois }\end{array}$ & 01 an & $\begin{array}{l}02 \\
\text { ans }\end{array}$ & matin & Midi & Soir \\
\hline $\begin{array}{l}\text { Pourcenta } \\
\text { ge }\end{array}$ & $\begin{array}{l}30,54 \\
\%\end{array}$ & $34,72 \%$ & $\begin{array}{l}24,11 \\
\%\end{array}$ & $\begin{array}{l}9,00 \\
\%\end{array}$ & $\begin{array}{l}1,61 \\
\%\end{array}$ & $\begin{array}{l}54,34 \\
\%\end{array}$ & $\begin{array}{l}12,54 \\
\%\end{array}$ & $\begin{array}{l}33,11 \\
\%\end{array}$ \\
\hline
\end{tabular}

C'est particulièrement en saison de pluies (39,54\%) que la Grue est la plus observée comparativement à la saison sèche froide (32,79\%) et la saison chaude sèche (27,65\%). 82,31\% des personnes ont rencontrées une colonie de 1 à 10 individus et seulement 1,92\% ont rencontrés de plus de 20 individus (Tableau 3).

Tableau 3. Proportion d'individus rencontrés par saison

\begin{tabular}{|l|l|l|l|l|l|}
\hline \multicolumn{5}{|l|}{ Saison de rencontre } & \multicolumn{4}{l|}{ Nombres rencontrés } \\
\hline Pluies & Sèches & Chaudes & 1 à 10 & 10 à 20 & Plus \\
\hline $39,54 \%$ & $32,79 \%$ & $27,65 \%$ & $82,31 \%$ & $15,75 \%$ & $1,92 \%$ \\
\hline
\end{tabular}

\section{Menaces sur l'espèce}

Les menaces qui pèsent sur cette espèce sont d'ordre naturel et/ou anthropique. La sècheresse $(87,13 \%)$ est la principale cause naturelle de la disparition de l'espèce tandis que les causes anthropiques les plus fréquemment citées sont la destruction de l'habitat $(38,26 \%)$ et la chasse (36,01\%) (Tableau 4)

Tableau 4. Proportion des menaces sur l'espèce

\begin{tabular}{|c|c|c|}
\hline Causes & Valeurs & Pourcentage \\
\hline \multicolumn{3}{|c|}{ Causes naturelles } \\
\hline Sécheresse & 271 & $87,13 \%$ \\
\hline Changement climatique & 25 & $8,03 \%$ \\
\hline Autres causes naturelles & 5 & $1,60 \%$ \\
\hline \multicolumn{2}{|c|}{ Causes anthropiques } \\
\hline Destruction de l'habitat & 119 & $38,26 \%$ \\
\hline Feu de brousse & 40 & $12,86 \%$ \\
\hline Chasse & 112 & $36,01 \%$ \\
\hline Pêche & 9 & $2,89 \%$ \\
\hline Agriculture & 11 & $3,53 \%$ \\
\hline Elevage & 13 & $4,18 \%$ \\
\hline Autres causes anthropiques & 7 & $2,25 \%$ \\
\hline
\end{tabular}

\section{Facteurs déterminants dans la distribution de l'espèce selon les enquêtés}

La distribution de l'espèce est déterminée par la destruction de son habitat selon 38,90\% des personnes enquêtées, alors que la végétation occupe seulement 1,92\% (Figure 2). 


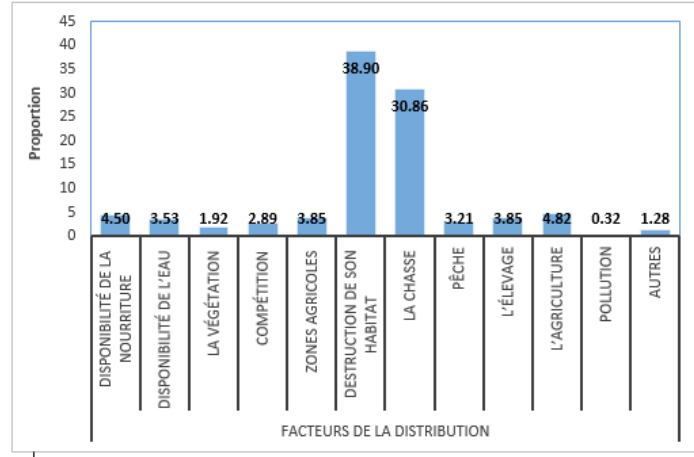

Figure 2. Facteurs déterminants dans la distribution de l'espèce

\section{Corrélation entre les causes anthropiques et les facteurs déterminants}

La corrélation entre les causes anthropiques et les facteurs déterminants dans la distribution de la Grue couronnée est forte ( $r=0,941$ et $p<0,05$ ) (figure 3).

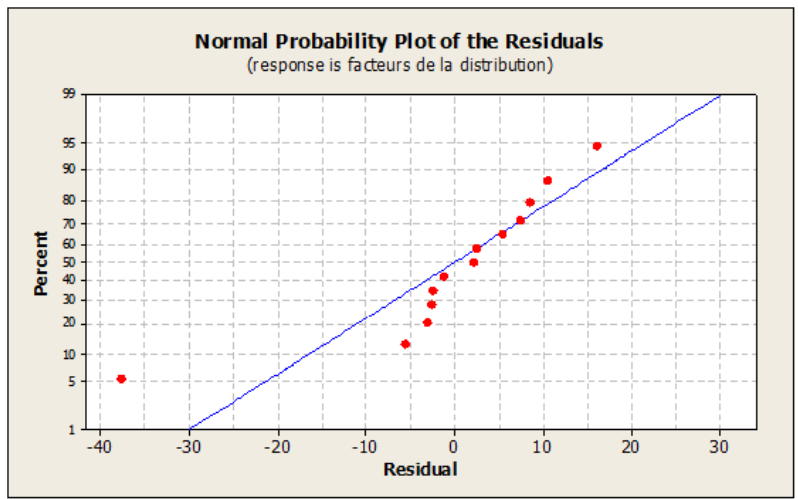

Figure 3. Droite de régression entre les causes anthropiques et les facteurs déterminants

\section{Régime alimentaire et mode d'alimentation de l'espèce}

Le tableau 5 montre que sur 51,76\% des personnes enquêtées, la Grue couronnée se nourrit des graines et 1,92\% par les autres types de nourriture. Selon les sondages, 55,30\% des populations sont favorables pour une abondance de nourriture contre 10,93\% pour une insuffisance en vivre.

Tableau 5. Sondage sur la nature et de la disponibilité de la nourriture

\begin{tabular}{|c|c|c|c|c|c|c|}
\hline \multicolumn{3}{|c|}{ Aliments } & \multicolumn{3}{c|}{ Disponibilité des aliments } \\
\hline Insectes & Graines & Poissons & Autres & abondante & $\begin{array}{c}\text { peu } \\
\text { abondante }\end{array}$ & pas abondante \\
\hline $33,11 \%$ & $51,76 \%$ & $13,18 \%$ & $1,92 \%$ & $55,30 \%$ & $33,76 \%$ & $10,93 \%$ \\
\hline
\end{tabular}

\section{Mode d'alimentation}

L'analyse de la figure 4 révèle que la Grue couronnée cherche sa nourriture en marchant selon $85 \%$ des personnes enquêtées. Elle cherche sa nourriture en pêchant (12\%) et en volant (3\%) selon les personnes enquêtées. 


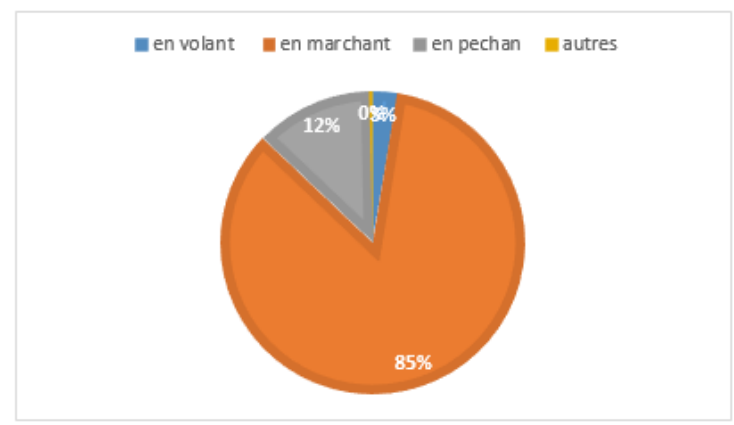

Figure 4. Mode d'alimentation de l'espèce

\section{Mode de reproduction de l'espèce}

\section{Caractérisation des œufs}

Le sondage des populations a montré que la Grue couronnée pond plus de deux œufs $(65,91 \%)$ et un œuf $(1,60 \%)$ (Tableau 6). Les œufs de $B$. pavonina sont blancs $(87,13 \%)$ selon les personnes enquêtées et les autres (12,87\%) ont mentionné une autre couleur (jaunâtre, bleue, verte). Les œufs de la Grue couronnée sont de grande taille selon 81,99\% des personnes enquêtées et de petite taille selon seulement $0,64 \%$ des personnes enquêtées (Tableau 6).

Tableau 6. Caractéristiques des œufs de la Grue couronnée

\begin{tabular}{|c|c|c|c|c|c|c|c|}
\hline \multicolumn{3}{|c|}{ Nombre des œufs } & \multicolumn{2}{c|}{ Couleur des oufs } & \multicolumn{3}{c|}{ Taille des oufs } \\
\hline Un & Deux & Plus & Blanche & Autres & Petite & $\begin{array}{c}\text { Moyenn } \\
\text { e }\end{array}$ & Grande \\
\hline $1,60 \%$ & $32,47 \%$ & $65,91 \%$ & $87,13 \%$ & $12,86 \%$ & $0,64 \%$ & $17,36 \%$ & $81,99 \%$ \\
\hline
\end{tabular}

\section{Lieu de ponte et incubation des œufs}

L'analyse du tableau 7 montre que la Grue couronnée pond ses œufs dans les jachères et au bord des eaux dans les proportions respectives de $64,95 \%$ et $5,46 \%$ de la population. Les nids sont construits au sol avec des débris de bois et d'herbe. La couvaison des œufs dure un mois $(83,92 \%)$ et une semaine $(0,32 \%)$ selon les propos recueillis.

Tableau 7. Lieu de ponte des œufs et durée d'incubation

\begin{tabular}{|c|c|c|c|c|c|c|}
\hline \multicolumn{3}{|c|}{ Lieu de ponte } & \multicolumn{3}{c|}{ Durée de la couvaison } \\
\hline $\begin{array}{c}\text { Au bord de } \\
\text { l'eau }\end{array}$ & $\begin{array}{c}\text { Dans les } \\
\text { champs }\end{array}$ & $\begin{array}{c}\text { Dans les } \\
\text { Jachères }\end{array}$ & $\begin{array}{c}\text { Une } \\
\text { semaine }\end{array}$ & Un mois & $\begin{array}{c}\text { Deux } \\
\text { mois }\end{array}$ & $\begin{array}{c}\text { Trois } \\
\text { mois }\end{array}$ \\
\hline $5,46 \%$ & $29,58 \%$ & $64,95 \%$ & $0,32 \%$ & $83,92 \%$ & $13,50 \%$ & $2,25 \%$ \\
\hline
\end{tabular}

\section{Eclosion des œufs et devenir des naissains}

L'analyse du tableau 8 montre que trois œufs de la Grue couronnée s'éclosent avec $71,06 \%$ contre un seul œuf (1,60\%) selon l'opinion des personnes enquêtées. Les sondages montrent que deux de ces oisillons 
arriveront à l'âge adulte $(53,37 \%)$ et rarement au-dessus de trois individus (2,89\%).

Tableau 8. Nombre d'œufs à l'incubation et devenir des naissains

\begin{tabular}{|c|c|c|c|c|c|c|c|c|}
\hline \multicolumn{4}{|c|}{ Taux d'éclosion } & \multicolumn{4}{c|}{ Des naissains à l’âge adulte } \\
\hline Zéro & Un & Deux & Trois & Plus & Un & Deux & Trois & Plus \\
\hline 0 & $1,60 \%$ & $18,00 \%$ & $71,06 \%$ & $9,32 \%$ & $17,68 \%$ & $53,37 \%$ & $26,04 \%$ & $2,89 \%$ \\
\hline
\end{tabular}

\section{Discussion}

La Grue couronnée comme la plupart des espèces aviaires a un moment d'activité le matin et le soir. Les colonies de l'espèce dans la zone ne dépassent pas plus de 10 individus et que l'espèce est présente dans la zone pendant toutes les saisons de l'année. Le faible nombre de l'espèce Balearica pavonina est dû à plusieurs menaces dont la chasse et la destruction de son habitat. Ces menaces ont une corrélation significative avec la distribution de la Grue couronnée. Selon Toudjani (2012), la chasse a un impact négatif sur les espèces d'oiseaux fréquentant la zone, conduisant ainsi à une réduction considérable du nombre d'espèces et d'individus d'oiseaux ces dernières années. Plusieurs études sur les espèces aviaires ont eu à évoquer ces menaces. Selon Hinda (2014), les agressions les plus dommageables subits par la Grue cendrée en Algérie sont celles qui interviennent sur les lieux même où doit se perpétuer l'espèce. L'importance de l'hivernage de la Grue cendrée s'est réduite ces dernières années, suite d'une part, à la sécheresse et d'autre part, à la construction de petits barrages et de petites retenues collinaires (Hinda, 2014). Dans l'Oranie, la plus occidentale des écorégions d'Algérie, les zones humides sont très polluées et constituent des déversoirs d'eaux usées en provenance des villes limitrophes telles qu'Oran. Aussi, la nature salée des sols ne permet pas la mise en cultures céréalières, altérant sérieusement l'installation hivernale de ces Grues (Isenmann et Moali, 2000). Dans cette même écorégion, dayet El-Ferd, entourée par de nombreuses surfaces céréalières, constitue toutefois un site privilégié de gagnage pour ce grand échassier granivore (Moulay, 2011). A Sétif en Algérie, la Grue cendrée est sujette, à une forte pression de braconnage bien que protégée (Baaziz et al., 2006 ; Houhamdi et al., 2010). Ce facteur est une source permanente de dérangement qui occasionne un effet immédiat de fuite et d'abondant des sites d'hivernage. Les effets conjugués de la pression anthropique et de la variabilité climatique (sécheresse accentuée) ne favorisent donc plus l'installation des Grues (Hinda, 2014). La diminution enregistrée des effectifs de Grues dans les hauts plateaux algériens (Houhamdi et al., 2010) coïncident avec l'augmentation démographique observée sur les sites habituels d'hivernage en Europe (Leito et al., 2006 ; Prange, 2010 et Nowald, 2010).

Cette étude montre que la Grue couronnée est une espèce omnivore qui 
se nourrit essentiellement des graines et des insectes. Plusieurs études sur les oiseaux ont eu à évoquer le régime alimentaire. Les oiseaux d'eau occupent au niveau des réseaux trophiques diverses positions (herbivores, zooplanctonophages, insectivores, piscivores) généralement situés au sommet des chaînes alimentaires et leur diversité nous renseigne sur le fonctionnement des divers milieux qu'ils occupent (Samraoui, 2009). De nombreuses études montrent l'importance des graines dans l'alimentation des canards (Brochet et al., 2009). Des espèces herbivores se nourrissent des tiges et des feuilles des plantes aquatiques (Amin, 2012). Il y a des espèces qui se nourrissent de proies animales. Toutes ingèrent des particules (sable, petits cailloux ou débris de coquilles de mollusques) pour faciliter le broyage mécanique de leur nourriture (Tamisier et Dehorter, 1999). Les oiseaux, de façon générale, sont capables de distinguer la nourriture parmi les autres particules par la vue et l'odorat (Martin, 2007 ; Steiger et al., 2008).

L'étude montre que la Grue couronnée est une espèce qui ponde 2 ou 3 œufs de grande taille et de couleur blanche. Cette pondaison se fait dans les jachères où la végétation constitue une protection pour les nids. La couvaison dure un mois. Aux moins 2 œufs de la Grue couronnée s'éclosent et 2 oisillons sont susceptibles d'arrivés à l'âge adulte. Plusieurs études sur les espèces aviaires (Grues et Canards) ont eu à mentionner ces aspects. La reproduction des oiseaux d'eau comprend de manière générale les phases de cantonnement et de formation des couples, de construction du nid, de ponte, d'incubation, d'éclosion, d'élevage, et d'envol des jeunes (Mohammed, 2016). Pour certaines espèces, il faut aussi ajouter après l'envol une période plus ou moins longue de dépendance des jeunes envers leurs parents (Tamisier et Dehoter, 1999). Plusieurs facteurs peuvent influencer la reproduction chez les oiseaux. La diminution du volume des œufs chez le grèbe castagneux (en Algérie) en fonction de la saison peut refléter une baisse des ressources alimentaires, mais la taille des œufs peut être influencée par une série de facteurs, y compris l'âge maternel, la qualité de la femelle et la taille de la grandeur de ponte (Fjeldsa, 2004). Selon Mohammed (2016), les différences dans la taille des œufs grèbe castagneux peuvent se rapporter à un gradient latitudinal ou des variations géographiques entre les populations distinctes. L'éclosion chez le grèbe castagneux est asynchrone, une éventuelle stratégie d'adaptation pour ajuster la taille des couvées à un environnement imprévisible (Amundsen et Slagvold, 1996) à travers les décès sélectifs des oisillons les plus jeunes, peutêtre par la famine, bien que le mécanisme exact est inconnu chez cette espèce. La prédation est connue pour constituer une cause majeure de l'échec des nids des oiseaux (Martin, 1993), alors que l'inondation peut également avoir un fort impact sur l'issue de l'élevage où le niveau d'eau fluctue (Mudge et Talbot, 1993). L'accès à de bons sites d'alimentation est essentiel pour maintenir l'état du corps pendant la saison d'élevage et de minimiser la prédation des nids en 
raison de l'absence momentanée des parents (Shoji et al., 2011). La reproduction du Grèbe huppé au lac Tonga (Algérie) peut être contrainte par la croissance de la végétation qui fournit la couverture de nidification et les ressources trophiques (Sarra, 2016).

\section{Conclusion}

La Grue couronnée figure parmi les espèces en voie de disparition. La Grue couronnée est grégaire et ne migre pas. Elle se rassemble en groupes plus ou moins importants selon les possibilités de nourrissage. La Grue couronnée est sédentaire et ne fait que quelques déplacements journaliers ou saisonniers. La Grue couronnée cherche sa nourriture tout en marchant. La saison de reproduction a lieu entre juillet et octobre. La femelle dépose 2 à 3 œufs dans un nid construit au sol. Les deux parents incubent pendant environ 28 à 31 jours.

En Afrique, les principaux facteurs causant le déclin des populations sont la perte d'habitat, la domestication et le commerce illégal des sujets en vie. On peut citer également la sècheresse, le changement climatique, la chasse et le feu de brousse. Cette étude vise à contribuer à la préservation de cette ressource animale. Ces résultats serviront de base de données pour une gestion rationnelle de cette espèce.

\section{References:}

1. Amin K. 2012. Etude du régime alimentaire hivernal des oiseaux d'eau en Numidie. Mémoire de Magister. Université du 08 Mai 1945, Guelma Algérie. 84p

2. Amundsen T. et Slagsvold T. 1996. Lack's brood reduction hypothesis and avian hatching asynchrony: what's next? Oikos, 76: 613-620

3. Baaziz N, Mayache B, Saheb M, Bensaci E, Ounissi M, Metallaoui S, Baldassarre G. et Bolen E. 2006. Waterfowl ecology and management, 2nd ed. Krieger Publ

4. Beilfuss R. D, Dodman T. et Urban E. K. 2007. The Status of Cranes in Africa in 2005. Ostrich: J. Afr. Ornithol. 78 (2):175-184.

5. Boere G. C, Galbraith C. A. et Stroud D. A. 2006. Water birds around the World. The Stationary office, Edinburgh, UK. P 960.

6. Brochet A, Guillemain M, Fritz H, Gauthier C. M. et Green A. 2009. The role of migratory ducks in the long-distance dispersal of native plants and the spread of exotic plants in Europe. Ecography 32: 919_928.

7. Cissé H. D. 2013. Intégration de la biodiversité dans l'évaluation environnementale stratégique des aménagements dans le bassin 
fluvial du programme Kandadji au Niger. Thèse de doctorat, Université du Québec à Montréal, Montréal, Québec, 331 p

8. Fjeldsa J. 2004. The grebes. Oxford University Press, Oxford

9. Fousseni H. 2000. Indicateurs d'existence des avantages potentiels d'une zone humide de type " $\mathrm{M}$ » : cas de la rivière Sara dans la préfecture d'Assoli au Togo. Mémoire de DESS, CRESA-Niamey, (Niger), 58 pages.

10. Geesing D. et Djibo H. 2006. Country Pasture/Forage Resource Profiles: Niger. In Food and Agriculture Organisation of the United Nations (FAO).

11. Georges K. 2015. Impacts des barrages sur les populations d'hippopotames et gestion du conflit avec l'homme : le cas du barrage de Kandadji sur le fleuve Niger. Mémoire de Maitre en Biologie, Université de SHERBROOKE. 81p.

12. Harris J. et Mirande C. 2013. A global overview of Cranes: Status, threats and conservation priorities, International Crane Foundation, Baraboo, USA. Les oiseaux de la Chine. 4(3):189-209.

13. Hinda H. 2014. Ecologie des Grues cendrées hivernants dans l'écocomplexe des zones humides des hautes plaines de l'Est algérien. Thèse de doctorat. Université du 08 Mai 1945, Guelma Algérie. 119p

14. Houhamdi M. 2011. Statut phénologique et reproduction des peuplements d'oiseaux d'eau dans l'éco-complexe de zones humides de Sétif (Hauts plateaux, Est de l'Algérie). Bulletin de l'Institut Scientifique de Rabat. 32(2): 77-87.

15. Houhamdi M, Baaziz N, Hafid H, Maazi M. C, Seddik S, Mayache B, Chefrour A. et Saheb M. 2010. Ecology of wintering Commons Cranes Grus grus in Algeria. $7^{\text {th }}$ European Crane Conference, (1417/10/2010). Stralsund. Allemagne.

16. Issiaka Y. 2004. Importance des zones humides du parc national du W du Niger pour les oiseaux d'eau migrateurs d'AfriqueEurasie.108p.

17. Issiaka Y. 2011. Importance des zones humides du parc national du $\mathrm{W}$ du Niger pour les oiseaux d'eau afrotropicaux et migrateurs du paléarctique occidental. Thèse, Université Abdou Moumouni de Niamey. 228p

18. Leito A, Keskpaik J, Ojaste I. et Truu J. 2006. The Eurasian Crane in Estonia.-Eesti Loodusfoto, Tartu.184p

19. Martin G. 2007. Visual fields and their functions in birds. J Ornithol 148:547-562.

20. Martin T. E. 1993 Nest predation and nest sites. BioScience, 43: 523532 
21. Mohammed A. 2016. Ecologie de la reproduction du Grèbe castagneux Tachybaptus ruficollis en Algérie. Thèse de doctorat. Université du 08 Mai 1945, Guelma Algérie. 152p

22. Moulay M. K. 2011. Analyse de la chronologie d'occupation de la zone humide Dayet El-Ferd par les oiseaux d'eau. Mémoire de magister. Université Aboubekr Belkaïd, Tlemcen, Algérie. 148p.

23. Mudge G. p. et Talbot T. R. 1993. The breeding biology and causes of nest failure of Scottish black-throated diver Gavia arctica. Ibis, 135: 113-120

24. Nowald G. 2010. Colour marking and radio traking of Common crane Grus grus in Germany and Europe an overview. Vogelwelt 131: 113-116.

25. Prange H. 2010. Migration and resting of the Common crane Grus grus and changes in four decades. Vogelwelt 131: 155-169.

26. Samraoui C. F. 2009. Contribution à l'étude de l'écologie de la reproduction des ardéidés (Héron Garde-bœufs Ardea ibis, Héron Crabier Ardeola ralloides, aigrette garzette Egretta garzetta et Héron bihoreau Nycticorax nycticorax) en Numidie Nord-Est algérien. Thèse. Université des sciences et de la technologie Houari Boumediene. 201p

27. Sarra C. 2016. Ecologie de la reproduction du Grèbe huppé Pdiceps cristatus au lac Tonga. Thèse de doctorat. Université du 08 Mai 1945, Guelma Algérie. 147p

28. Shoji A, Elliott K. H, Aris B. S, Crump D. et Gaston A. J. 2011. Incubation patterns in a central-place forager affect lifetime reproductive success: scaling of patterns from a foraging bout to a lifetime. PLoS ONE, 6: e17760.

29. Sinsin B. et Kampman D. 2010. Atlas de la biodiversité de l'Afrique de l'ouest. Tome 1 : Benin. Cotonou et frunkfurt/main. 726p

30. Soumaïla H. 2014. Impact de l'urbanisation sur la distribution et la diversité aviaire de la ville de Niamey. Mémoire Master2 BGESSS, Université de Maradi. 64p

31. Steiger S, Fidler A, Valcu M. et Kempenaers B. 2008. Avian olfactory receptor gene repertoires: evidence for a well-developed sense of smell in birds? Proc R Soc B Biol Sci 275:2309-2317.

32. Tamisier A. et Dehorter O. 1999. Camargue, canards et foulques. Fonctionnement d'un prestigieux quartier d'hiver. Centre Ornithologique du Gard, Nîmes.

33. Tecsult (Tecsult International Limitée). 2006a. Programme Kandadji de Régénération des Écosystèmes et de Mise en valeur de la vallée du Niger : Étude d'impact environnemental et social détaillée. In Worldbank. 
34. Tecsult (Tecsult International Limitée). 2006b. Chapitre 5 : Description du milieu biophysique. Rapports définitifs de la Phase I : Description du Milieu (Volume 1). Montréal, Tecsult International Limitée, 92 p.

35. Toudjani A. 2012. Contribution à la typologie de la faune aviaire des zones humides de la région de Maradi, Niger : cas des mares de Madarounfa. Mémoire Master 2 BGESSS, Université de Maradi. 90p 